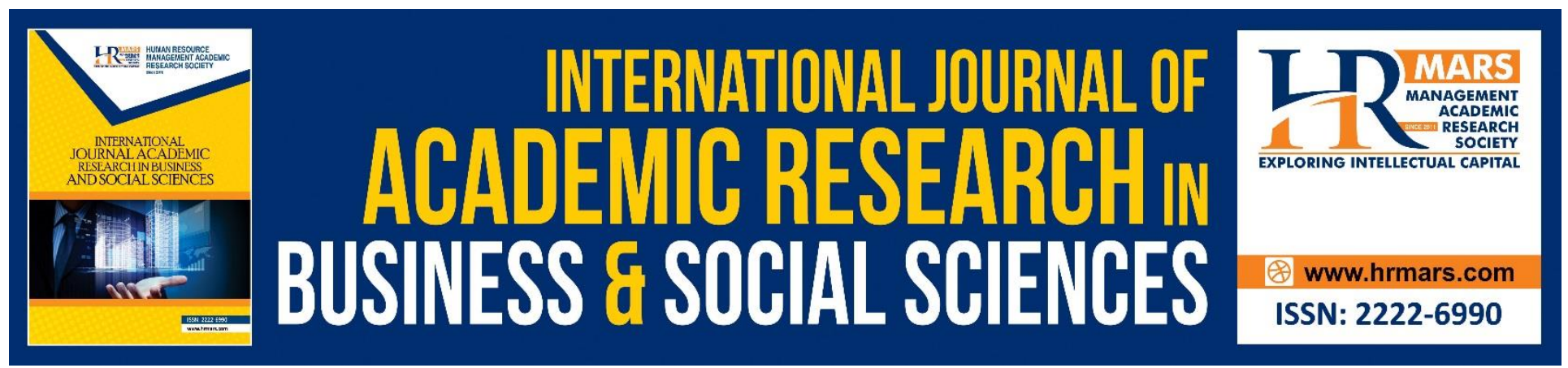

\title{
Performance Assessment Standard Approach Based on Form 1 Physical Education Secondary School Curriculum Standard
}

\begin{abstract}
Norkhalid Salimin, Julismah Jani, Sanmuga Nathan Jeganathan \& Mohd Izwan Shahril
\end{abstract}

To Link this Article: http://dx.doi.org/10.6007/IJARBSS/v9-i6/5926

DOI: $10.6007 /$ IJARBSS/v9-i6/5926

Received: 02 April 2019, Revised: 17 May 2019, Accepted: 1 June 2019

Published Online: 26 June 2019

In-Text Citation: (Salimin, Jani, Jeganathan, \& Shahril, 2019)

To Cite this Article: Salimin, N., Jani, J., Jeganathan, S. N., \& Shahril, M. I. (2019). Performance Assessment Standard Approach Based on Form 1 Physical Education Secondary School Curriculum Standard Norkhalid Salimin, Julismah Jani, Sanmuga Nathan Jeganathan \& Mohd Izwan Shahril. International Journal of Academic Research in Business and Social Sciences, 9(6), 105-117.

Copyright: (C) 2019 The Author(s)

Published by Human Resource Management Academic Research Society (www.hrmars.com)

This article is published under the Creative Commons Attribution (CC BY 4.0) license. Anyone may reproduce, distribute, translate and create derivative works of this article (for both commercial and non-commercial purposes), subject to full attribution to the original publication and authors. The full terms of this license may be seen

at: http://creativecommons.org/licences/by/4.0/legalcode

Vol. 9, No. 6, 2019, Pg. $105-117$

http://hrmars.com/index.php/pages/detail/IJARBSS

JOURNAL HOMEPAGE

Full Terms \& Conditions of access and use can be found at http://hrmars.com/index.php/pages/detail/publication-ethics 


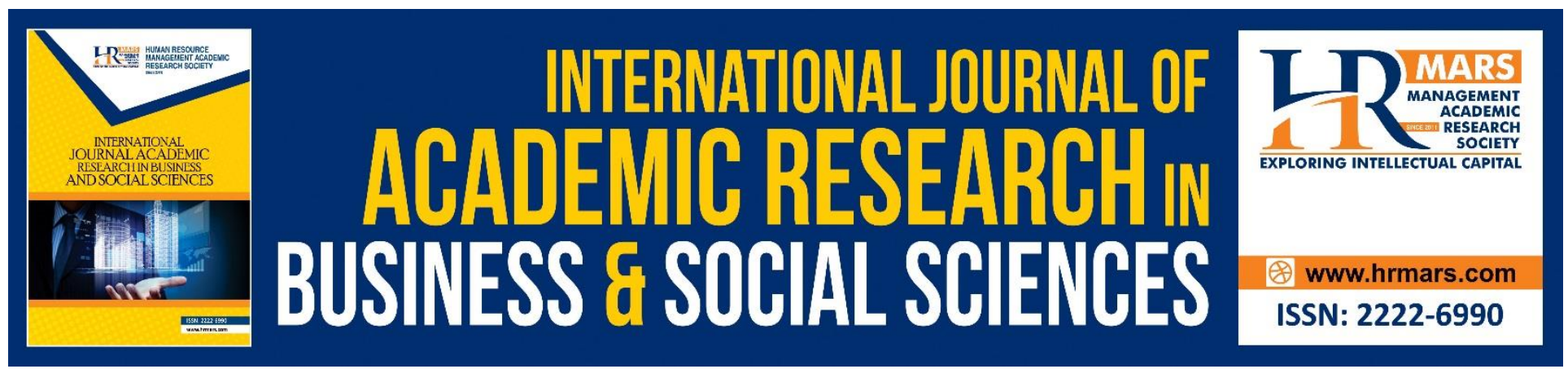

\title{
Performance Assessment Standard Approach Based on Form 1 Physical Education Secondary School Curriculum Standard
}

\section{Norkhalid Salimin, Julismah Jani, Sanmuga Nathan Jeganathan \& Mohd Izwan Shahril}

Faculty of Sports Science and Coaching, Sultan Idris Education University

Email: norkhalid@fsskj.upsi.edu.my

\begin{abstract}
The purpose of this study was to develop a standard school-based assessment module in Physical Education to evaluate the performance assessment standard of Form 1 students. In general, the research was conducted to develop and evaluate the effectiveness of the Performance Assessment Standard Approach (PASA) Module in Form 1 Secondary School Standard Curriculum. Preexperimental study design was used in this study in three schools in Muslim district, Perak consisted of 235 Form 1 students using Form 1 Physical Education Student Performance Standard instrument form (validity; $r=.79$ \& reliability; $r=.71$ ). In overall, the achievement level of learning performance standards for learning aspects based on PASA for Unit 2 - Rhythmic Movement, Unit 4 - Volleyball, Unit 5 - Basic Athletics, Unit 6 - Recreation and Leisure were Good for all items, namely Thinking Strategies, Reasoning Skills, Critical Thinking Skills and Creative Thinking Skills. The findings also showed that there is a significant relationship between student achievement levels by learning unit. The study concluded that PASA could identify the achievement level holistically and comprehensively. The implication of the study suggested PASA instrument to be used as an alternative instrument to determine the student learning achievement level based on higher order thinking skills.
\end{abstract}

Keyword: Assessment, Physical Education, Higher Order Thinking Skills.

\section{Introduction}

In 2017, Secondary School Integrated Curriculum has been transformed into the Secondary School Standard Curriculum (KSSM). The transformation is a reinforcement and enhancement to the Secondary School Integrated Curriculum (KBSM) in terms of organization, content, pedagogy, time allocation, assessment method, material and curriculum management in schools (Ministry of Education, 2012). Accordingly, the Statement of Professional Circular No. 2/2013 dated December 16, 2013 (Ministry of Education Malaysia, 2013) notes that from 2017, KSSM is implemented in stages starting from Form 1 using the Standard Document for Curriculum and Assessment (DSKP). 
The guidelines in Malaysian Education Blueprint 2013-2025 are to improve the assessment framework for adding items and testing higher order thinking skills as well as moving towards using standards in School Based Assessment (Ministry of Education, 2012). The implementation of SBA in schools is fully implemented by teachers by planning, administering, giving scores and reporting the assessment in accordance with the procedures set by the Malaysian Examinations Board (Ministry of Education, 2012). Hence, SBA is the catalyst for the strengthening of the nation's education system in the development of human capital to realize the goal of Secondary School Standard Curriculum, Malaysian Education Development Plan and National Education Philosophy.

School assessment is not just an assessment of learning achievement after the completion of a learning process but should also include formative assessments throughout the learning process so that students can improve learning achievement (Torrance, 2007). The assessment of learning process includes a) assessment of learning b) assessments as learning and c) assessment for learning so that students are given the opportunity to collect data on the understanding and mastery of their learning through self-assessment and peer assessment (Earl, 2012).

Matanin and Tannehill (1994) report that teachers agreed that the assessment process for students in Physical Education should be carried out continuously, formally, systematically and objectively. This is because the assessment is carried out with the aim of motivating students as well as detecting their weaknesses. Furthermore, assessment can also play a role as a response to teachers' teaching as well as helping teachers organize activities so that students benefit from the teaching and learning (Miller, 2006). However, the study of Othman, Esah, Ahmad Fuaad, Azali, and Hisham (2013) state that Physical Education and Health Education teachers were still inefficient in developing test items, unclear about the meaning of valid and reliable assessments, less skilful in assessing the outcome of the assessment. This situation occurs because the teacher still does not have the appropriate assessment competency to carry out the assessment effectively (Othman et al., 2013). Subsequently, the implementation of Physical Education curriculum was comparable to other subjects and was not implemented half-heartedly at school (Munira, 2010).

In that regard, assessment in Physical Education subjects should include assessments of cognitive, psychomotor and affective domains that focus on Higher Order Thinking Skills (HOTS). Therefore, the researchers intend to construct a standard school-based assessment module in Physical Education to assess the performance assessment standards of Form 1 students. The Performance Assessment Standard Approach (PASA) Module in this study involves assessment criteria for Teaching and Facilitating referring to Form 1 Physical Education Standard Document for Curriculum and Assessment (DSKP) based on Secondary School Standard Curriculum (KSSM) and Form 1 Physical Education and Health Education textbook.

Based on the research aims stated above, several research objectives have been developed as follows: 
INTERNATIONAL JOURNAL OF ACADEMIC RESEARCH IN BUSINESS AND SOCIAL SCIENCES Vol. 9, No. 6, June, 2019, E-ISSN: 2222-6990 @ 2019 HRMARS

i. To identify the value of validity and reliability of the Performance Assessment Standard Approach (PASA) Module in Physical Education subject based on Form 1 Secondary School Standard Curriculum.

ii. To identify the achievement levels of student learning performance standard in learning aspects in Form 1 Physical Education based on Performance Assessment Standard Approach (PASA) Module.

iii. To investigate the relationship between the achievements of student learning performance standard in different learning aspects in Form 1 Physical Education based on Performance Assessment Standard Approach (PASA) Module.

\section{Methodology}

The design of this study is a pre-experimental one-shot case study. The design is used because it is appropriate for educational studies as used in the study by Norkhalid et al., (2017), Izwan, Norkhalid, and Gunathevan (2015). As shown in Figure 1, the conceptual framework for the construction of Performance Assessment Standard Approach (PASA) Module based on Form 1 Physical Education KSSM is based on the Instructional Design Model (Dick \& Carey, 2001). The flow of the study process involves three categories of schools based on Form 1 Physical Education DSKP. Data analysis based on the Student Performance Assessment Standard is obtained based on the teacher's assessment.

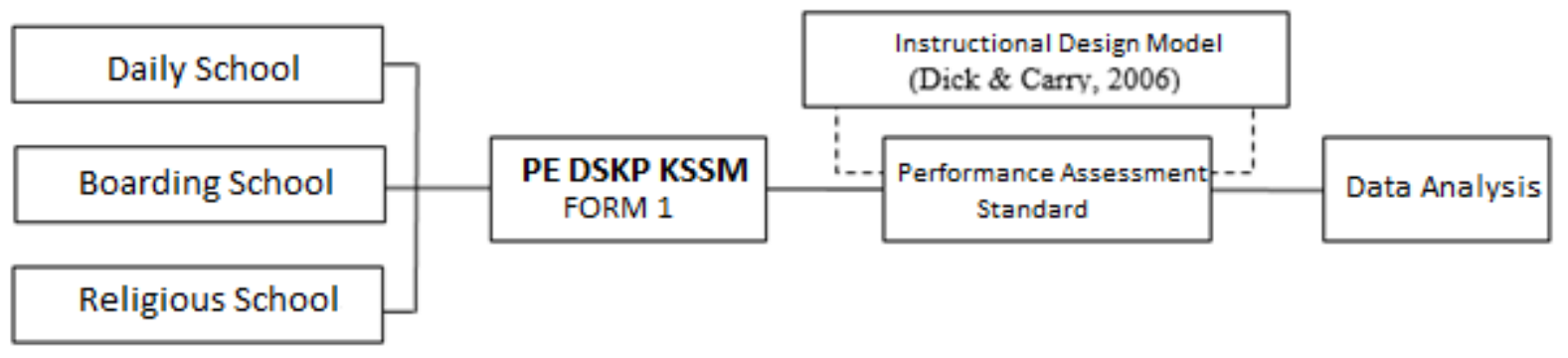

Figure 1. Research Conceptual Framework

This study involved 235 Form 1 students and 6 Form 1 Physical Education teachers of Batang Padang District in Perak. This study was conducted using a self-developed research instrument, i.e. Form 1 Physical Education Student Performance Standard Form. ADDIE model (Rossett, 1987) and Morrow et al. (2005) model were used in constructing Performance Assessment Standard based on Form 1 Physical Education Secondary School Standard Curriculum. The researchers used the guidance instruments construction by Morrow et al. (2005) in the process of constructing items in Performance Assessment Standard based on Form 1 Physical Education KSSM in development stage.

\section{Findings and Discussions}

The research instrument constructed was submitted to 3 expert panels (content, implementer (teacher), language) using content evaluation form to evaluate the validity of content $(r=0.87)$ while test and re-test method was used to obtain instrument reliability during pilot study. 
INTERNATIONAL JOURNAL OF ACADEMIC RESEARCH IN BUSINESS AND SOCIAL SCIENCES

Vol. 9, No. 6, June, 2019, E-ISSN: 2222-6990 @ 2019 HRMARS

Table 1. The Validity of Performance Assessment Standard Approach (PASA) Module based on Form 1 Physical Education Standard Curriculum Secondary School $(\mathrm{N}=6)$

\begin{tabular}{ccccc}
\hline Item & Content & Executor & Language & Overall \\
\hline & Expert 1 & Expert 2 & Expert 3 & \\
Item 1 & 9 & 10 & 8 & \\
Item 2 & 8 & 9 & 5 & \\
Item 3 & 8 & 10 & 3 & \\
Item 4 & 9 & 9 & 7 & \\
Item 5 & 9 & 9 & 7 & .79 \\
$M$ & 0.86 & 0.94 & 0.60 & \\
\hline
\end{tabular}

Based on Table 1, the validity value of Performance Assessment Standard Approach Module in Form 1 Physical Education subject based on Secondary School Standard Curriculum in pilot study was .79 $(n=3)$. According to Sidek and Jamaludin (2005), the value of .70 is considered to have mastered or achieved high achievement levels.

Table 2. The Reliability Value of Performance Assessment Standard Approach (PASA) Module based on Form 1 Physical Education Standard Curriculum Secondary School $(N=35)$

\begin{tabular}{llc}
\hline \multicolumn{1}{c}{ Variable } & Analysis Technique & $r$ \\
\hline (Unit 2 Rhythmic Movements) Thinking Strategies & Pearson product moment & $0.828^{*}$ \\
(Unit 2 Rhythmic Movements) Reasoning Skills & Pearson product moment & $0.857^{*}$ \\
(Unit 2 Rhythmic Movements) Critical Thinking Skills & Pearson product moment & $0.828^{*}$ \\
(Unit 2 Rhythmic Movements) Creative Thinking Skills & Pearson product moment & $0.717^{*}$ \\
(Unit 4 Volleyball) Thinking Strategies & Pearson product moment & $0.751^{*}$ \\
(Unit 4 Volleyball) Reasoning Skills & Pearson product moment & $0.795^{*}$ \\
(Unit 4 Volleyball) Critical Thinking Skills & Pearson product moment & $0.606^{*}$ \\
(Unit 4 Volleyball) Creative Thinking Skills & Pearson product moment & $0.795^{*}$ \\
(Unit 5 Basic Athletics) Thinking Strategies & Pearson product moment & $0.799^{*}$ \\
(Unit 5 Basic Athletics) Reasoning Skills & Pearson product moment & $0.736^{*}$ \\
(Unit 5 Basic Athletics) Critical Thinking Skills & Pearson product moment & $0.406^{*}$ \\
(Unit 5 Basic Athletics) Creative Thinking Skills & Pearson product moment & $0.753^{*}$ \\
(Unit 6 Recreation \& Leisure) Thinking Strategies & Pearson product moment & $0.676^{*}$ \\
(Unit 6 Recreation \& Leisure) Reasoning Skills & Pearson product moment & $0.520^{*}$ \\
(Unit 6 Recreation \& Leisure) Critical Thinking Skills & Pearson product moment & $0.535^{*}$ \\
(Unit 6 Recreation \& Leisure)) Creative Thinking Skills & Pearson product moment & $0.723^{*}$ \\
& & $r=.71$ \\
\hline
\end{tabular}

*Significance level at 0.05

Table 2 showed that the reliability coefficient of Performance Assessment Standard Approach (PASA) Module based on Form 1 Physical Education Standard Curriculum Secondary School for 15 items was between .41 to $.83(\mathrm{M}=.71, \mathrm{~N}=35)$. The interpretation of the reliability coefficient that can be adopted according to research practitioners in social sciences is greater than $r=.60$. According to 
INTERNATIONAL JOURNAL OF ACADEMIC RESEARCH IN BUSINESS AND SOCIAL SCIENCES Vol. 9, No. 6, June, 2019, E-ISSN: 2222-6990 @ 2019 HRMARS

Mohd Majid (2000) the level of $r=.71$ to .99 is the acceptable item reliability. Based on the findings, the reliability value of the PASA Module in Physical Education subject under Form $1 \mathrm{KSSM}$ is high and acceptable.

Table 3 showed the achievement level of student learning performance for learning aspects in Form 1 Physical Education based on Performance Assessment Standard Approach (PASA) Module for Unit 2 - Rhythmic Movement. There are two activities in Unit 2 - Rhythmic Movement namely Rise Together and Joget and Sumazau. The finding indicated that in overall, the students achieved Good level for all items in Rise Together activity, that is Thinking Strategies (55.50\%, $n=131$ ), Reasoning Skills (58.10\%, $n=137)$, Critical Thinking Skills $(55.50 \%, n=131)$ and Creative Thinking Skills $(53.80 \%$, $\mathrm{n}=127)$. However, for the Joget and Sumazau activity, the overall achievement of the students was at the Mastery level, namely Thinking Strategies $(42.80 \%, n=101)$, Reasoning Skills $(51.30 \%, n=121)$, Critical Thinking Skills $(52.10 \%, n=123)$ and Creative Thinking Skills $(56.80 \%, n=134)$.

Table 3. Student Achievement Level Unit 2 - Rhythmic Movement

\begin{tabular}{|c|c|c|c|c|c|c|c|}
\hline Item & E & G & $\mathrm{M}$ & LM & VLM & Min & SD \\
\hline \multicolumn{8}{|c|}{ Unit 2: Rhythmic Movement Activity: Rise Together } \\
\hline Thinking Strategies & $\begin{array}{c}1 \\
(0.40)\end{array}$ & $\begin{array}{c}131 \\
(55.50)\end{array}$ & $\begin{array}{c}96 \\
(40.70)\end{array}$ & $\begin{array}{c}7 \\
(3.00)\end{array}$ & - & 3.54 & $\begin{array}{c}0.5 \\
4\end{array}$ \\
\hline Reasoning Skills & $\begin{array}{c}4 \\
(1.70)\end{array}$ & $\begin{array}{c}137 \\
(58.10)\end{array}$ & $\begin{array}{c}91 \\
(38.60)\end{array}$ & $\begin{array}{c}3 \\
(1.30)\end{array}$ & - & 3.66 & $\begin{array}{c}0.5 \\
2\end{array}$ \\
\hline Critical Thinking Skills & $\begin{array}{c}5 \\
(2.10)\end{array}$ & $\begin{array}{c}131 \\
(55.50)\end{array}$ & $\begin{array}{c}97 \\
(41.10)\end{array}$ & $\begin{array}{c}2 \\
(0.80)\end{array}$ & - & 3.59 & $\begin{array}{c}0.5 \\
5\end{array}$ \\
\hline $\begin{array}{l}\text { Creative Thinking } \\
\text { Skills }\end{array}$ & $\begin{array}{c}4 \\
(1.70)\end{array}$ & & & $\begin{array}{c}4 \\
(1.70)\end{array}$ & - & 3.55 & $\begin{array}{c}0.5 \\
6\end{array}$ \\
\hline \multicolumn{8}{|c|}{ Unit 2: Rhythmic Movement - Joget and Sumazau } \\
\hline Thinking Strategies & $\begin{array}{c}8 \\
(3.40)\end{array}$ & $\begin{array}{c}118 \\
(50.00)\end{array}$ & $\begin{array}{c}101 \\
(42.80)\end{array}$ & $\begin{array}{c}8 \\
(3.40)\end{array}$ & - & 3.54 & $\begin{array}{c}0.6 \\
2\end{array}$ \\
\hline Reasoning Skills & $\begin{array}{c}5 \\
(2.10)\end{array}$ & $\begin{array}{c}99 \\
(41.90)\end{array}$ & $\begin{array}{c}121 \\
(51.30)\end{array}$ & $\begin{array}{c}10 \\
(4.20)\end{array}$ & - & 3.42 & $\begin{array}{c}0.6 \\
1\end{array}$ \\
\hline Critical Thinking Skills & $\begin{array}{c}2 \\
(0.80)\end{array}$ & $\begin{array}{c}103 \\
(43.60)\end{array}$ & $\begin{array}{c}123 \\
(52.10)\end{array}$ & $\begin{array}{c}7 \\
(3.0)\end{array}$ & - & 3.43 & $\begin{array}{c}0.5 \\
7\end{array}$ \\
\hline $\begin{array}{l}\text { Creative Thinking } \\
\text { Skills }\end{array}$ & $\begin{array}{c}2 \\
(0.80)\end{array}$ & $\begin{array}{c}92 \\
(39.00)\end{array}$ & $\begin{array}{c}134 \\
(56.80)\end{array}$ & $\begin{array}{c}7 \\
(3.00)\end{array}$ & - & 3.38 & $\begin{array}{c}0.5 \\
6\end{array}$ \\
\hline
\end{tabular}

Table 4 showed the achievement level of student learning performance for learning aspects in Form 1 Physical Education based on Performance Assessment Standard Approach (PASA) Module for Unit 4 - Volleyball. There are two activities in Unit 4 - volleyball, i.e. Digging Champion and Setting Rally. The findings showed that the achievement of the students as a whole is at the Mastery level for all the items in Digging Champion activity, i.e. Thinking Strategies (44.90\%, $n=106)$, Reasoning Skills $(48.70 \%, n=115)$, Critical Thinking Skills $(51.70 \%, n=122)$ and Creative Thinking Skills $(51.70 \%, n=122)$. 
INTERNATIONAL JOURNAL OF ACADEMIC RESEARCH IN BUSINESS AND SOCIAL SCIENCES

Vol. 9, No. 6, June, 2019, E-ISSN: 2222-6990 @ 2019 HRMARS

Meanwhile, for Setting Rally activity, the students achieved Mastery level with Thinking Strategies (64.40\%, $n=152)$, Reasoning Skills $(68.20 \%, n=161)$, Critical Thinking Skills $(67.80 \%, n=160)$ and Creative Thinking Skills $(65.30 \%, \mathrm{n}=154)$.

Table 4. Student Achievement Level Unit 4 - Volleyball

\begin{tabular}{|c|c|c|c|c|c|c|c|}
\hline Item & $E$ & G & $\mathrm{M}$ & LM & VLM & Min & SD \\
\hline \multicolumn{8}{|c|}{ Unit 4: Volleyball - Activity: Digging Champion } \\
\hline Thinking Strategies & $\begin{array}{c}1 \\
(0.40)\end{array}$ & $\begin{array}{c}115 \\
(48.70)\end{array}$ & $\begin{array}{c}106 \\
(44.90)\end{array}$ & $\begin{array}{c}13 \\
(5.50)\end{array}$ & - & 3.44 & $\begin{array}{c}0.6 \\
1\end{array}$ \\
\hline Reasoning Skills & $\begin{array}{c}3 \\
(1.30)\end{array}$ & $\begin{array}{c}104 \\
(44.10)\end{array}$ & $\begin{array}{c}115 \\
(48.70)\end{array}$ & $\begin{array}{c}13 \\
(5.50)\end{array}$ & - & 3.41 & $\begin{array}{c}0.6 \\
2\end{array}$ \\
\hline Critical Thinking Skills & $\begin{array}{c}1 \\
(0.40)\end{array}$ & $\begin{array}{c}100 \\
(42.40)\end{array}$ & $\begin{array}{c}122 \\
(51.70)\end{array}$ & $\begin{array}{c}12 \\
(5.10)\end{array}$ & - & 3.38 & $\begin{array}{c}0.5 \\
9\end{array}$ \\
\hline $\begin{array}{l}\text { Creative Thinking } \\
\text { Skills }\end{array}$ & $\begin{array}{c}1 \\
(0.40)\end{array}$ & $\begin{array}{c}98 \\
(41.50)\end{array}$ & $\begin{array}{c}122 \\
(51.70)\end{array}$ & $\begin{array}{c}14 \\
(5.90)\end{array}$ & - & 3.36 & $\begin{array}{c}0.6 \\
0\end{array}$ \\
\hline \multicolumn{8}{|c|}{ Unit 4: Volleyball - Setting Rally } \\
\hline Thinking Strategies & - & $\begin{array}{c}68 \\
(28.80)\end{array}$ & $\begin{array}{c}152 \\
(64.40)\end{array}$ & $\begin{array}{c}15 \\
(6.40)\end{array}$ & - & 3.23 & $\begin{array}{c}0.5 \\
5\end{array}$ \\
\hline Reasoning Skills & - & $\begin{array}{c}60 \\
(25.40)\end{array}$ & $\begin{array}{c}161 \\
(68.2)\end{array}$ & $\begin{array}{c}14 \\
(5.90)\end{array}$ & - & 3.20 & $\begin{array}{c}0.5 \\
3\end{array}$ \\
\hline Critical Thinking Skills & - & $\begin{array}{c}61 \\
(25.80)\end{array}$ & $\begin{array}{c}160 \\
(67.80)\end{array}$ & $\begin{array}{c}14 \\
(5.90)\end{array}$ & - & 3.20 & $\begin{array}{c}0.5 \\
3\end{array}$ \\
\hline $\begin{array}{l}\text { Creative Thinking } \\
\text { Skills }\end{array}$ & - & $\begin{array}{c}70 \\
(29.70)\end{array}$ & $\begin{array}{c}154 \\
(65.30)\end{array}$ & $\begin{array}{c}11 \\
(4.70)\end{array}$ & - & 3.25 & $\begin{array}{c}0.5 \\
3\end{array}$ \\
\hline
\end{tabular}

E-Excellent; G-Good; M-Mastery; LM-Lack Mastery, VLM-Very Lack Mastery

Table 5 showed the achievement level of student learning performance for learning aspects in Form 1 Physical Education based on Performance Assessment Standard Approach (PASA) Module for Unit 5 - Basic Athletics. There are two activities in Unit 5 - Basic Athletics, Fosbury Flop Style High Jump and Parry O'Brien Style Shot Put. The findings showed that the achievement of students as a whole was at a Good level for all items in the Fosbury Flop Style High Jump activity, namely Thinking Strategies $(51.30 \%, n=121)$, Reasoning Skills $(50.00 \%, n=118)$, Critical Thinking Skills $(54.70 \%=129)$ and Creative Thinking Skills $(62.30 \%, n=147)$. Whereas Parry O'Brien Style Shot Put activity, the students achieved Good level, i.e. Thinking Strategies (42.80\%, $n=101)$, Reasoning Skills $42.40 \%, n=$ 92), Critical Thinking Skills $(39.00 \%, n=192)$ and Creative Thinking Skills $(54.20 \%, n=128)$. 
INTERNATIONAL JOURNAL OF ACADEMIC RESEARCH IN BUSINESS AND SOCIAL SCIENCES Vol. 9, No. 6, June, 2019, E-ISSN: 2222-6990 (C) 2019 HRMARS

Table 5. Student Achievement Level Unit 5 - Basic Athletics

\begin{tabular}{|c|c|c|c|c|c|c|c|}
\hline Item & $\mathrm{E}$ & G & M & LM & VLM & Min & SD \\
\hline \multicolumn{8}{|c|}{ Unit 5: Basic Athletics- Activity: Fosbury Flop Style High Jump } \\
\hline Thinking Strategies & - & $\begin{array}{c}121 \\
(51.30)\end{array}$ & $\begin{array}{c}85 \\
(36.00)\end{array}$ & $\begin{array}{c}29 \\
(12.30)\end{array}$ & - & 3.39 & $\begin{array}{c}0.7 \\
0\end{array}$ \\
\hline Reasoning Skills & - & $\begin{array}{c}118 \\
(50.00)\end{array}$ & $\begin{array}{c}96 \\
(40.70)\end{array}$ & $\begin{array}{c}21 \\
(8.90)\end{array}$ & - & 3.41 & $\begin{array}{c}0.6 \\
5\end{array}$ \\
\hline $\begin{array}{l}\text { Critical Thinking } \\
\text { Skills }\end{array}$ & - & $\begin{array}{c}129 \\
(54.70)\end{array}$ & $\begin{array}{c}89 \\
(37.70)\end{array}$ & $\begin{array}{c}17 \\
(7.20)\end{array}$ & - & 3.48 & $\begin{array}{c}0.6 \\
3\end{array}$ \\
\hline $\begin{array}{l}\text { Creative Thinking } \\
\text { Skills }\end{array}$ & - & $\begin{array}{c}147 \\
(62.30)\end{array}$ & $\begin{array}{c}73 \\
(30.90)\end{array}$ & $\begin{array}{c}15 \\
(6.40)\end{array}$ & - & 3.56 & $\begin{array}{c}0.6 \\
1\end{array}$ \\
\hline \multicolumn{8}{|c|}{ Unit 5: Basic Athletics- Activity: Parry O’Brien Style Shot Put } \\
\hline Thinking Strategies & $\begin{array}{c}7 \\
(3.00)\end{array}$ & $\begin{array}{c}101 \\
(42.80)\end{array}$ & $\begin{array}{c}103 \\
(43.60)\end{array}$ & $\begin{array}{c}24 \\
(10.20)\end{array}$ & - & 3.39 & $\begin{array}{c}0.7 \\
1\end{array}$ \\
\hline Reasoning Skills & $\begin{array}{c}6 \\
(2.50)\end{array}$ & $\begin{array}{c}100 \\
(42.40)\end{array}$ & $\begin{array}{c}107 \\
(45.30)\end{array}$ & $\begin{array}{c}22 \\
(9.30)\end{array}$ & - & 3.38 & $\begin{array}{c}0.6 \\
9\end{array}$ \\
\hline $\begin{array}{l}\text { Critical Thinking } \\
\text { Skills }\end{array}$ & $\begin{array}{c}6 \\
(2.50)\end{array}$ & $\begin{array}{c}92 \\
(39.00)\end{array}$ & $\begin{array}{c}125 \\
(53.00)\end{array}$ & $\begin{array}{c}12 \\
(5.10)\end{array}$ & - & 3.39 & $\begin{array}{c}0.6 \\
3\end{array}$ \\
\hline $\begin{array}{l}\text { Creative Thinking } \\
\text { Skills }\end{array}$ & $\begin{array}{c}3 \\
(1.30)\end{array}$ & $\begin{array}{c}128 \\
(54.20)\end{array}$ & $\begin{array}{c}93 \\
(39.40)\end{array}$ & $\begin{array}{c}11 \\
(4.70)\end{array}$ & - & 3.52 & $\begin{array}{c}0.6 \\
1\end{array}$ \\
\hline
\end{tabular}

E-Excellent; G-Good; M-Mastery; LM-Lack Mastery, VLM-Very Lack Mastery

Table 6 showed the achievement level of student learning performance for learning aspects in Form 1 Physical Education based on Performance Assessment Standard Approach (PASA) Module for Unit 6 - Recreation and Leisure. There are two activities in Unit 6 - Recreation and Leisure, i.e. Spider-Web and Alligator Swamp. The results showed that the achievement of the students as a whole was at Good level for all items in Spider-Web activity, i.e. Thinking Strategies (64.00\%, $n=151)$, Reasoning Skills (60.60\%, $n=143$, Critical Thinking Skills $(62.30 \%, n=147)$ and Creative Thinking Skills (66.90\%, $\mathrm{n}=158)$. The overall performance of the students is at a good level for Alligator Swamp activity, namely Thinking Strategies (64.00\%, $n=151)$, Reasoning Skills $(57.60 \%, n=136)$, Critical Thinking Skills (61.90\%, $n=146)$ and Creative Thinking Skills (63.60\%, $n=150)$. 
INTERNATIONAL JOURNAL OF ACADEMIC RESEARCH IN BUSINESS AND SOCIAL SCIENCES Vol. 9, No. 6, June, 2019, E-ISSN: 2222-6990 (C) 2019 HRMARS

Table 6. Student Achievement Level Unit 6 - Recreation and Leisure

\begin{tabular}{|c|c|c|c|c|c|c|c|}
\hline Item & $E$ & $\mathrm{G}$ & $M$ & LM & VLM & Min & SD \\
\hline \multicolumn{8}{|c|}{ Unit 6: Recreation and Leisure- Activity: Spider-Web } \\
\hline \multirow[t]{2}{*}{ Thinking Strategies } & 3 & 151 & 79 & 2 & - & 3.65 & 0.5 \\
\hline & $(1.30)$ & $(64.00)$ & $(33.50)$ & $(0.80)$ & & & 2 \\
\hline \multirow[t]{2}{*}{ Reasoning Skills } & 5 & 143 & 76 & 11 & - & 3.60 & 0.6 \\
\hline & (2.10) & $(60.60)$ & $(32.20)$ & $(4.70)$ & & & 1 \\
\hline Critical Thinking & 1 & 147 & 76 & 11 & - & 3.59 & 0.5 \\
\hline Skills & $(0.40)$ & $(62.30)$ & $(32.20)$ & $(4.70)$ & & & 9 \\
\hline Creative Thinking & 2 & 158 & 64 & 11 & - & 3.64 & 0.5 \\
\hline Skills & $(0.80)$ & $(66.90)$ & $(27.10)$ & $(4.70)$ & & & 8 \\
\hline \multicolumn{8}{|c|}{ Unit 6: Recreation and Leisure- Activity: Alligator Swamp } \\
\hline \multirow[t]{2}{*}{ Thinking Strategies } & 2 & 151 & 60 & 22 & - & 3.56 & 0.6 \\
\hline & $(0.80)$ & $(64.00)$ & $(25.40)$ & (9.30) & & & 7 \\
\hline \multirow[t]{2}{*}{ Reasoning Skills } & 4 & 136 & 65 & 30 & - & 3.49 & 0.7 \\
\hline & (1.70) & $(57.60)$ & $(27.50)$ & (12.70) & & & 4 \\
\hline Critical Thinking & 1 & 146 & 67 & 21 & - & 3.54 & 0.6 \\
\hline Skills & $(0.40)$ & (61.90) & $(28.40)$ & (8.90) & & & 6 \\
\hline Creative Thinking & 1 & 150 & 63 & 21 & - & 3.56 & 0.6 \\
\hline Skills & $(0.40)$ & $(63.60)$ & $(26.70)$ & $(8.90)$ & & & 6 \\
\hline
\end{tabular}

E-Excellent; G-Good; M-Mastery; LM-Lack Mastery, VLM-Very Lack Mastery

Table 7 showed the correlation between student achievement levels by learning unit. The findings showed that there is a significant relationship between Unit 2: Rhythmic Movement with Unit 4: Volleyball $(r=.55)$, Unit 2: Rhythmic Movement with Unit 5: Basic Athletics $(r=.43)$, Unit 2: Rhythmic Movement with Unit 6: Recreation and Leisure $(r=.35)$, Unit 4: Volleyball with Unit 5: Basic Athletics $(r=.48)$, Unit 4: Volleyball with Unit 6: Recreation and Leisure $(r=.59)$ and Unit 5: Basic Athletics with Unit 6 - Recreation and Leisure $(r=.39)$. 
INTERNATIONAL JOURNAL OF ACADEMIC RESEARCH IN BUSINESS AND SOCIAL SCIENCES Vol. 9, No. 6, June, 2019, E-ISSN: 2222-6990 @ 2019 HRMARS

Table 7. Relationship of Student Achievement Levels by Learning Unit

\begin{tabular}{|c|c|c|c|c|c|}
\hline & & Unit 2 & Unit 4 & Unit 5 & Unit 6 \\
\hline \multirow[t]{3}{*}{$\begin{array}{l}\text { Uni } \\
\text { t } 2\end{array}$} & $\begin{array}{l}\text { Pearson } \\
\text { Correlation }\end{array}$ & 1 & $.548^{* *}$ & $.429^{* *}$ & $.347^{* *}$ \\
\hline & Sig. (2-tailed) & & .000 & .000 & .000 \\
\hline & $\mathrm{N}$ & 235 & 233 & 235 & 235 \\
\hline \multirow[t]{3}{*}{$\begin{array}{l}\text { Uni } \\
\text { t } 4\end{array}$} & $\begin{array}{l}\text { Pearson } \\
\text { Correlation }\end{array}$ & $.548^{* *}$ & 1 & $.481^{* *}$ & $.594^{* *}$ \\
\hline & Sig. (2-tailed) & .000 & & .000 & .000 \\
\hline & $\mathrm{N}$ & 233 & 233 & 233 & 233 \\
\hline \multirow[t]{3}{*}{$\begin{array}{l}\text { Uni } \\
\text { t } 5\end{array}$} & $\begin{array}{l}\text { Pearson } \\
\text { Correlation }\end{array}$ & $.429^{* *}$ & $.481^{* *}$ & 1 & $.389^{* *}$ \\
\hline & Sig. (2-tailed) & .000 & .000 & & .000 \\
\hline & $\mathrm{N}$ & 235 & 233 & 235 & 235 \\
\hline \multirow[t]{3}{*}{$\begin{array}{l}\text { Uni } \\
\text { t } 6\end{array}$} & $\begin{array}{l}\text { Pearson } \\
\text { Correlation }\end{array}$ & $.347^{* *}$ & $.594^{* *}$ & $.389^{* *}$ & 1 \\
\hline & Sig. (2-tailed) & .000 & .000 & .000 & \\
\hline & $\mathrm{N}$ & 235 & 233 & 235 & 235 \\
\hline
\end{tabular}

**. Significance level at 0.01 (2-tailed).

The achievement level of student learning performance standard for learning aspects in Form 1 Physical Education based on Performance Assessment Standard Approach (PASA) Module for Unit 2 - Rhythmic Movement, Unit 4 - Volleyball, Unit 5 - Basic Athletics, Unit 6 - Recreation and Leisure as a whole was at Good level for all items, i.e. Thinking Strategies, Reasoning Skills, Critical Thinking Skills and Creative Thinking Skills. The findings also showed that there was a significant relationship between the levels of student achievement by learning unit.

Assessment in Physical Education is one of the issues most frequently discussed among Physical Education practitioners over 40 years (López-pastor, Kirk, Lorente, Macphail, \& Macdonald, 2013). Studies show that Physical Education teachers use various forms of assessment to assess students' performance. In grading the achievement of students, Physical Education teachers not only use motor skills and fitness levels but also the criteria of effort, participation, student behaviors (Matanin \& Tannehill, 1994; Melograno, 2007) citizenship, sportsmanship and preparations (Melograno, 2007) are also taken into account in giving grades to student achievement. However, such assessments are actually very subjective in grading and this situation actually indicates the failure of Physical Education teachers in assessing and documenting student achievement (Julismah, Norkhalid, Mohd Izwan \& Kamaruzaman, 2014).

The achievement levels of Form 1 student learning based on Performance Assessment Standard Approach (PASA) Module include the thinking strategies, reasoning skills, critical thinking skills and creative thinking skills. Accordingly, Wee (2003) further affirms the importance of assessment. He 
INTERNATIONAL JOURNAL OF ACADEMIC RESEARCH IN BUSINESS AND SOCIAL SCIENCES Vol. 9, No. 6, June, 2019, E-ISSN: 2222-6990 @ 2019 HRMARS

noted that in Physical Education curriculum, there are two main purposes of assessment, i.e. assessment as feedback on student learning and assessment as the determinant of the effectiveness of the teaching of Physical Education teachers. As such, teachers need to ensure that student assessment activities are meaningful and beneficial to be carried out in line with the teaching and learning process (Sani, 2003). Formative assessment can be used to obtain information on the progress of a teaching process and summative assessment can be used if the teacher wishes to determine the grade or student achievement at the end of the lesson (Sani, 2003).

Fullan, (1993) stresses that in order to make important changes in the assessment system which could result in wider changes in the curriculum and pedagogy, we should encourage thoughtful reflection of how changes in the assessment need to be aligned with the curriculum's content choices, pedagogical decisions and what is seen as learning goal as a whole in learning teaching tasks or experiences. The findings also support Ravikumar, Ghani \& Aziah (2015) in a study aimed at identifying teachers' views on autonomy practices and assessment practices as well as values held on assessments at Cluster School Malaysia. The results of the study show that there is a significant positive relationship between teacher autonomy and assessment practice as well as assessment value.

\section{Conclusion}

Physical Education is part of the educational experience available at school. Learning aspects of psychomotor, cognitive and affective and focus on Higher Order Thinking Skills. In general, the research was conducted to develop and evaluate the effectiveness of the Performance Assessment Standard Approach (PASA) Module in Form 1 Physical Education based on Secondary School Standard Curriculum. The significance of this study was to evaluate the level of learning and student development by focusing on and emphasizing the 21st century skills acquisition. It is hoped that PASA will be an innovation of standard assessment model for learning and assessment of learning that teachers can apply in Form 1 PE. With this alternative assessment, the goal of the Ministry of Education Malaysia through $4^{\text {th }}$ Cluster Malaysian Education Blueprint 2013-2025 on the need for designing a range of school-based assessment tools to evaluate students will be achieved.

\section{Acknowledgement}

This study was part of research funded by the Sultan Idris Education University

(Code: GPUPend/ 2018-0058-107-01).

\section{References}

Sani, A. Y. (2003). Mengurus Sekolah: Siri Pentadbiran Pendidikan. Pahang: PTS Publications.

Dick, C. W. L. \& Carey, J. (2001). The systematic design of instruction (5th ed.). Allyn \& Bacon.

Earl, L. M. (2012). Assessment as learning: Using classroom assessment to maximize student learning. Corwin Press.

Fullan, M. G. (1993). Why Teachers Must Become Change Agents. Educational Leadership, 50(6), 1217. 
INTERNATIONAL JOURNAL OF ACADEMIC RESEARCH IN BUSINESS AND SOCIAL SCIENCES Vol. 9, No. 6, June, 2019, E-ISSN: 2222-6990 @ 2019 HRMARS

Julismah, J., Norkhalid, S., Izwan, M. S., \& Kamaruzaman, S. A. (2014). Model-model Pentaksiran dalam Pendidikan Jasmani (1st Ed.). Tanjong Malim, Perak: Universiti Pendidikan Sultan Idris.

Ministry of Education Malaysia (2012). Laporan Awal Pelan Pembangunan Pendidikan Malaysia 2013 - 2025. Kementerian Pelajaran Malaysia. Putrajaya.

Ministry of Education Malaysia (2013). Surat Pekeliling Ikhtisas Bil. 2 Tahun 2013: Pelaksanaan Kurikulum Standard Sekolah Rendah (KSSR) Tahap II Secara Berperingkat-peringkat Mulai Tahun 2014. Putrajaya.

López-pastor, V. M., Kirk, D., Lorente-, E., Macphail, A., \& Macdonald, D. (2013). Alternative assessment in physical education : a review of international literature. Sport Education and Society, 18(1), 57-76.

Matanin, M. \& Tannehill, D. (1994). Assessment and Grading in Physical Education. Journal of Teaching in Physical Education, 13, 395-405.

Melograno, V. J. (2007). Grading and Report Cards for Standards-Based Physical Education. Journal of Physical Education, Recreation \& Dance, 78(6), 45-53.

https://doi.org/10.1080/07303084.2007.10598041

Miller, K. D. (2006). Measurement by Physical Educator. Why and How. Singapore: Mc Graw Hill.

Izwan, M. S., Norkhalid, S., \& Gunathevan, E. (2015). The Validity and Reliability of ISO Test towards the Performance Assessment of Future Physical Education Teachers in Teaching and Learning Process. Procedia - Social and Behavioral Sciences, 195(December), 814-820. https://doi.org/10.1016/j.sbspro.2015.06.184

Konting, M. M. (2000). Kaedah penyelidikan pendidikan. Kuala Lumpur: Dewan Bahasa dan Pustaka. Morrow, J. R., Jackson, A. W., Disch, J. G., \& Mood, D. P. (2005). Measurement and evaluation in human performance (3rd ed.). Champaign, IL: Human Kinetics.

Munira, M. (2010). Pelaksanaan Kurikulum Pendidikan Jasmani oleh Guru Tingkatan Satu (Tesis Doktor Falsafah yang tidak diterbitkan). Universiti Malaya, Malaysia.

Norkhalid, S., Izwan, M. S., Julismah, J., Azali, R., Gunathevan, E., Liza, S., \& Akmaniah, N. (2017). Development Assessment Instrument for Basic Skills of Invasion in Physical Education. Adv. Sci. Lett. 23(1), 386-389.

Othman, L., Esah, S. M., Fuaad, A. D., Azali, R., \& Hisham, O. B. (2013). Pembinaan Standard Pentaksiran Berasaskan Sekolah bagi sekolah rendah di Malaysia. Tanjong Malim, Malaysia.

Ravikumar, V., Ghani, A. K. A., \& Aziah, I. (2015). The effect of teacher autonomy on assessment practices among Malaysian cluster schoolteachers. International Journal of Asian Social Science, 5 (1), 31-36.

Rossett, A. (1987). Training Needs Assessment. Englewood Cliffs: Educational Technology Publications.

Noah, S. M. \& Ahmad, J. (2005). Pembinaan Modul Bagaimana Membina Modul Latihan Dan Modul Akademik. Penerbit UPM: Serdang, Selangor.

Torrance, H. (2007). Assessment as learning? How the use of explicit learning objectives, assessment criteria and feedback in post-secondary education and training can come to dominate learning. 1. Assessment in Education: Principles, Policy \& Practice, 14(3), 281-294. https://doi.org/10.1080/09695940701591867 
INTERNATIONAL JOURNAL OF ACADEMIC RESEARCH IN BUSINESS AND SOCIAL SCIENCES Vol. 9, No. 6, June, 2019, E-ISSN: 2222-6990 @ 2019 HRMARS

Wee, E. H. (2003). Attitude of secondary school teachers toward physical education. Journal of Malaysian Association for Physical Education, Sport Science and Fitness, 3, 71-98. 